\title{
Oficinas de Elaboração de Comunicação e Escrita Científica com Estudantes Universitários
}

Workshops on the Development of Scientific Communication and Writing with College Students

Talleres de Elaboración de Comunicación y Escrita Científica con Estudiantes Universitarios
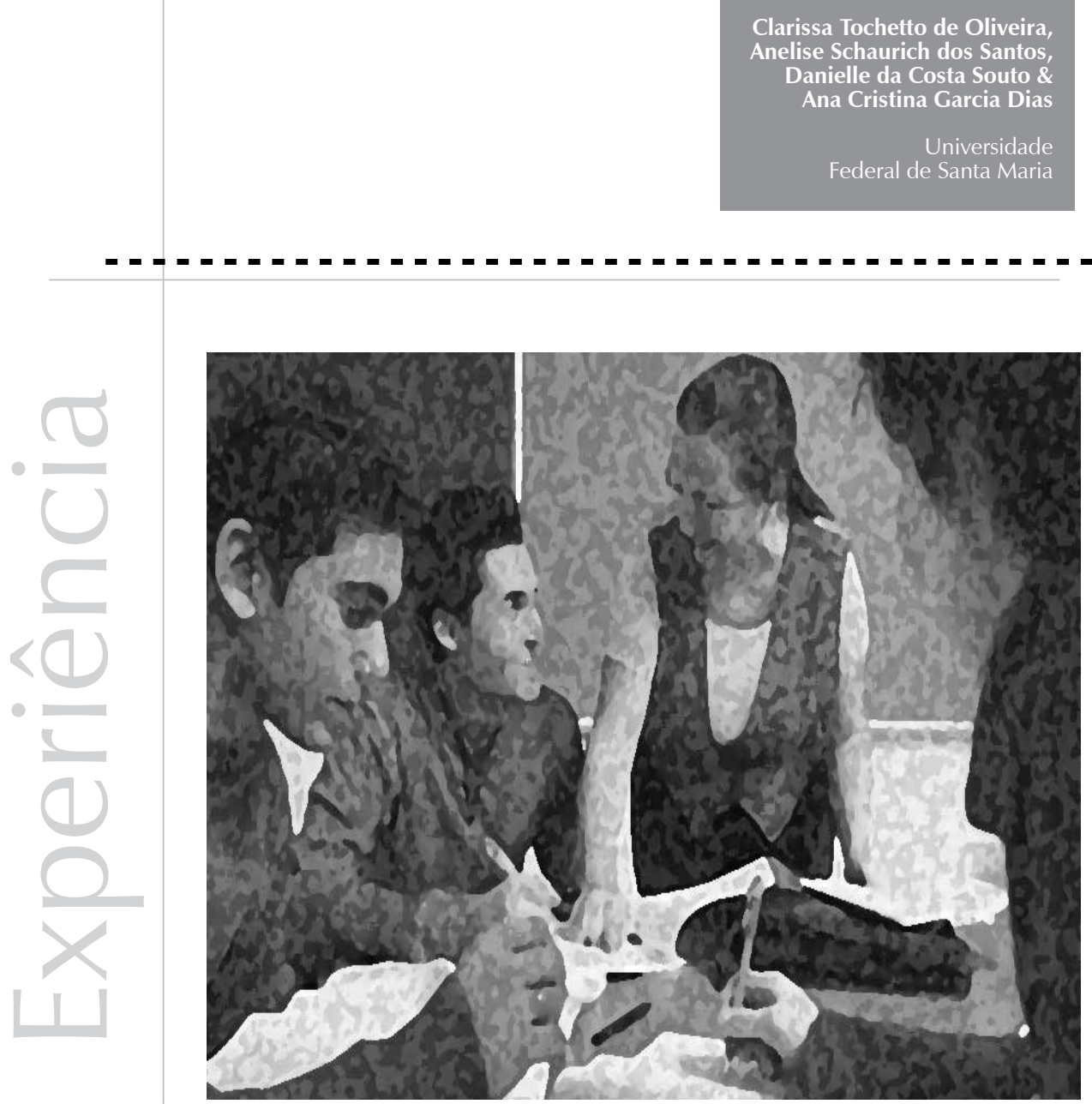
Resumo: Este estudo descreve uma experiência de oficinas de elaboração de trabalhos científicos realizadas com estudantes universitários de uma universidade pública do Rio Grande do Sul. Foram realizados três encontros com o objetivo de caracterizar as diversas modalidades de trabalhos científicos (resumo simples, resumo expandido, trabalho completo e artigo) e de orientar sobre os aspectos que devem ser considerados na sua elaboração. No total, participaram 44 alunos de graduação e pós-graduação. Os resultados possibilitaram a identificação das dúvidas dos discentes em relação ao tema abordado assim como à classificação de resumos em simples ou expandido, a forma como se elabora um problema de pesquisa e à metodologia. Essas dificuldades revelam lacunas na formação desses indivíduos, que suscitam questões sobre quais tópicos têm sido trabalhados nas disciplinas de metodologia da pesquisa, se e de que forma os grupos de pesquisa têm abordado esse tema ou ainda se acadêmicos de Iniciação Científica, mestrandos e doutorandos têm recebido instruções básicas de seus orientadores para a escrita científica. Conclui-se que as universidades poderiam ampliar os espaços para que esse tipo de conhecimento seja construído por meio da manutenção de oportunidades tradicionais e da criação de ações inovadoras, como é o exemplo dessa oficina.

Palavras-chave: Estudantes universitários. Oficina criativa. Comunicação científica. Habilidades para escrita.

Abstract: This study describes an experience with workshops on the elaboration of scientific work with college students from a university in Rio Grande do Sul. Three workshops were held in order to characterize different types of scientific work (abstract, extended abstract, short paper and full paper) and to explain some aspects that must be considered when writing these works. Forty four undergraduate and postgraduate students took part in the workshops. Procedures and tools used in the meetings were described. Results enabled the identification of students' questions regarding the subject, such as the classification of an abstract, the development of a research problem, and questions regarding methodology. These difficulties reveal fragilities of the background of these students, raising questions about what issues have been studied in research methodology courses, whether and how research groups have approached this subject, and/or if undergraduate research, master's and doctoral students have received basic instructions from their advisors for scientific writing. We conclude that the universities may widen activities so that this kind of knowledge can be built through the maintenance of traditional opportunities and the proposal of innovative actions, such as this workshop.

Keywords: College students. Creative workshop. Scientific communication. Writing skills.

Resumen: Este estudio describe una experiencia de talleres de elaboración de trabajos científicos, realizados con estudiantes universitarios de una universidad pública de Río Grande del Sur. Fueron realizados tres encuentros con el objetivo de caracterizar las diversas modalidades de trabajos científicos (resumen simple, resumen expandido, trabajo completo y artículo), y guiar sobre los aspectos que deben ser considerados en la elaboración de los mismos. Al total, participaron 44 alumnos de posgrado. Fueron descritos los procedimientos y las herramientas utilizadas. Los resultados posibilitaron la identificación de las dudas de los dicentes respecto al tema abordado, como clasificación de resúmenes simple o expandido, de cual manera se construye un problema de investigación y dudas sobre metodología. Esas dificultades demuestran huecos en la formación de esos individuos, que evocan cuestiones sobre cuales los temas han sido trabajados en las asignaturas de metodología de investigación, si y de qué manera los Grupos de Investigación han enfocado ese tema o todavía si académicos de Iniciación Científica, estudiantes de maestría y doctorado han recibido instrucciones básicas de sus tutores para la escrita científica. Se concluye que las universidades podrían expandir los espacios para que ese tipo de conocimiento sea construido por medio de la manutención de oportunidades tradicionales y de la creación de acciones innovadoras, así como el ejemplo de este taller. Palabras-clave: Estudiantes universitarios. Taller creativo. Comunicacion científica. Habilidades para escribir.

A participação em atividades de pesquisa faz parte da trajetória acadêmica de muitos estudantes de graduação. De acordo com o Conselho Nacional de Desenvolvimento Científico e Tecnológico (CNPq, 2002), as atividades de iniciação científica (IC) buscam introduzir o aluno no mundo da pesquisa, geralmente com a concessão de bolsas aos alunos de graduação como estímulo.
Assim, o ensino superior busca construir um espírito científico através da aprendizagem decorrente da participação nessas atividades (Machado, 2002), de forma que os discentes compreendam o que significa se tornar um pesquisador e como participar da cultura científica da instituição na qual está inserido (Hurtado et al., 2011). As atividades de IC caracterizam-se como um instrumento de 
De fato, alunos envolvidos em pelo menos uma atividade acadêmica

frequentemente relatam estar mais satisfeitos com a escolha profissional do que discentes que não as possuem (Bardagi, Lassance, Paradiso, \& Menezes, 2006). apoio teórico e metodológico à realização de um projeto de pesquisa e constituem um canal adequado de auxílio para a formação de uma nova mentalidade no aluno. A IC pode ser definida como um instrumento de formação (Maia, 2008) que pretende incentivar talentos potenciais entre os discentes, mediante participação em projetos de pesquisa, orientados por um pesquisador qualificado, a fim de prepará-los para o ingresso na pós-graduação (CNPq, 2002).

A experiência de IC também pode interferir na carreira do discente, uma vez que possibilita maior compreensão do mercado de trabalho por permitir a interação entre o mundo científico (teorias e fundamentos) e as atividades práticas da profissão (Reis, 2007). As atividades acadêmicas, como IC e a participação em eventos, também proporcionam maior envolvimento com o curso (Hurtado et al., 2011), e costumam ser associadas a maior identidade profissional, ou seja, a maior satisfação quanto à profissão escolhida (Bardagi, Lassance, \& Paradiso, 2003). De fato, alunos envolvidos em pelo menos uma atividade acadêmica frequentemente relatam estar mais satisfeitos com a escolha profissional do que discentes que não as possuem (Bardagi, Lassance, Paradiso, \& Menezes, 2006).

A discussão sobre a importância das atividades de pesquisa e da construção de trabalhos científicos no ensino superior é fundamental para os alunos que querem seguir a carreira de pesquisadores e docentes. Os trabalhos científicos são, em grande parte, produzidos por docentes e discentes no meio acadêmico (Machado, 2002). A publicação dos estudos permite a reflexão de outros investigadores sobre os resultados obtidos por determinado autor, uma vez que esse dissemina seu conhecimento e autoriza aos demais o acesso a sua produção (Sabadini, Sampaio, \& Koller, 2009). As publicações fazem com que a informação circule, o que, por sua vez, possibilita a socialização das novas descobertas em grande escala, viabilizando uma produção cumulativa e evitando esforços desnecessários ocasionados pelo simples desconhecimento do que já foi feito (Machado, 2002). Assim, pode-se afirmar que a divulgação das pesquisas científicas contribui para o avanço da ciência (Nahas, Ferreira, Neto, \& Garcia, 2004). Os estudos desenvolvidos podem ser apresentados em encontros científicos, como congressos, e na publicação de livros ou de artigos em periódicos. Muitos desses já podem ser encontrados na internet, que passou a ser um importante meio de divulgação das pesquisas (Nahas et al., 2004). A partir disso, constata-se a relevância da habilidade da escrita para o desempenho acadêmico e para a produção do conhecimento, bem como a importância da informação sobre os procedimentos de elaboração de dissertações, teses e monografias (Machado, 2002; Kellogg \& Raulerson, 2007).

A cobrança por publicações e pelo desenvolvimento de atividades de pesquisa é crescente, principalmente para quem pretende seguir a carreira docente e atuar como pesquisador. O número de trabalhos publicados, sua qualidade e o tipo de veículo publicitário são elementos fundamentais para a construção de um currículo sólido. Ademais, o destaque profissional decorrente do trabalho científico pode ser percebido nos incentivos que os pesquisadores recebem, por meio de bolsas e de outros auxílios financeiros, que evidenciam o interesse do Governo e de várias instituições de fomento à pesquisa no desenvolvimento científico (Petroianu, 2002). Contudo, tem-se observado que estudantes de diferentes graus de formação (graduação ou pós-graduação) ainda apresentam fragilidades no que diz respeito à aquisição de conhecimentos, de habilidades e de atitudes elementares à realização da pesquisa científica no ensino superior (Machado, 2002). 
Há professores que não explicam suas expectativas sobre a forma de escrever aos alunos, não os orientam, nem supervisionam seus trabalhos com comentários para que os estudantes possam compreender que aspectos podem ser melhorados no futuro (Carlino, 2007).
Parece ainda existir uma lacuna na formação básica dos acadêmicos para pesquisa no que se refere a leituras de textos científicos, à construção e à organização de projetos ou de textos acadêmicos, dentre outros. Essas lacunas são observadas tanto nos cursos de graduação quanto na pós-graduação (especialização, mestrado e doutorado). Além disso, o fato de o aluno gostar da atividade científica não pressupõe que ele apresente um bom preparo como pesquisador. $\mathrm{O}$ aluno pode ser apenas um curioso sobre os instrumentos teóricos e metodológicos de sua área e pode acontecer também que orientadores já não estejam dispostos a percorrer os caminhos que são pré-requisitos à elaboração de trabalhos acadêmicos e científicos a cada novo processo de orientação. Dessa forma, o conjunto de conhecimentos, habilidades e atitudes necessárias às atividades acadêmicas, por vezes, são tomados como supostamente aprendidos e geram dificuldades no processo de execução (Carlino, 2007; Giordani, Mendes, \& Henriques, 2007). Um exemplo disso é a própria habilidade da escrita, cujo desenvolvimento requer não só as instruções oferecidas pelo orientador mas também o treinamento da ortografia, da gramática, da pontuação, da organização das ideias e da coesão textual (Kellogg \& Raulerson, 2007). Há professores que não explicam suas expectativas sobre a forma de escrever aos alunos, não os orientam, nem supervisionam seus trabalhos com comentários para que os estudantes possam compreender que aspectos podem ser melhorados no futuro (Carlino, 2007). É evidente, então, a importância de atividades que possam complementar as lacunas deixadas nos diferentes níveis de ensino.

O objetivo deste artigo consiste em descrever uma experiência com oficinas de elaboração de trabalhos científicos realizadas com estudantes universitários de uma universidade pública no interior do Rio Grande do Sul. Essas oficinas buscavam orientar os discentes sobre as diferenças entre as modalidades de trabalhos científicos (resumo, resumo expandido, trabalho completo e artigo) e sobre os aspectos que devem ser considerados na sua elaboração.

\section{Método}

O projeto de extensão Oficinas de Orientação Profissional e Planejamento de Carreira oferece oficinas para estudantes universitários com o objetivo de contribuir para o desenvolvimento de habilidades relevantes para o planejamento de suas carreiras. Dentre as oficinas oferecidas nos anos 2011 e 2012, três abordaram a elaboração de trabalhos científicos em parceria com a Pró-Reitoria de Assuntos Estudantis PRAE (setor que trata de apoio aos estudantes da instituição). Esse setor é responsável pela divulgação, pela lista de inscrição, pelo fornecimento do local onde as oficinas são realizadas e pelos materiais necessários (como data-show, lista de mapeamento e demais instrumentos característicos de cada oficina). A iniciativa de oferecer tais atividades surgiu da identificação de dificuldades, nos relatos de estudantes nos atendimentos psicoterápicos realizados pelo setor psicossocial da PRAE, referentes à escrita científica e à elaboração de comunicações científicas, que estavam associados a problemas encontrados na vida universitária. Além desse fato, reconhece-se a importância de desenvolver essas habilidades para aumentar o comprometimento dos alunos com a formação, como já apontado pela literatura (Teixeira \& Gomes, 2004).

A divulgação das oficinas foi realizada através da página virtual da universidade, da página da PRAE e do projeto no Facebook, e por correio eletrônico. Os universitários interessados em participar entraram em contato, através do correio eletrônico indicado na notícia, para ter o seu nome incluído na lista de participantes. Em geral, as oficinas foram realizadas com os 20 primeiros estudantes que manifestaram interesse em delas participar no momento de divulgação e de inscrição. 
No total, 77 estudantes inscreveram-se, embora apenas 44 tenham comparecido. A idade dos estudantes universitários variou de 17 a 57 anos, sendo a média de 26 anos, e a mediana, de 23 anos. Os participantes eram alunos dos cursos de Administração (1 estudante), Artes Visuais (1 estudante), Ciências Sociais (4 estudantes), Direito (1 estudante), Economia (3 estudantes), Educação Física (2 estudantes), Educação Especial (3 estudantes), Engenharia da Computação (1 estudante), Engenharia de Controle e Automação (2 estudantes), Engenharia de Produção (1 estudante), Engenharia Mecânica (1 estudante), Farmácia (1 estudante), Filosofia (1 estudante), Letras (4 estudantes), Matemática (1 estudante), Pedagogia (4 estudantes), Produção Editorial (1 estudante), Tecnologia em Alimentos (1 estudante), Tecnologia em Geoprocessamento (4 estudantes) e Terapia Ocupacional (3 estudantes). Os estudantes que compareceram às oficinas estavam cursando, na sua maioria, os semestres iniciais e intermediários de seus cursos, embora também houvesse alunos dos semestres finais. A oficina ainda contou com a participação de alunos do curso Técnico Administrativo (1 estudante), de pós-graduação de Arquivologia (1 estudante), Design (1 estudante) e de Geografia (1 estudante). Zelou-se por trabalhar com 20 participantes, no máximo, por oficina, a fim de assegurar a efetividade das atividades desenvolvidas (poder tirar as dúvidas, abrir espaço para a participação). As oficinas foram elaboradas e ministradas por estudantes de graduação e de pós-graduação em Psicologia, orientados por um professor do mesmo departamento.

Os encontros foram realizados nas dependências da própria universidade, em horário que possibilitasse a participação de alunos com aulas diurnas e noturnas, e tiveram a duração de uma hora e trinta minutos, aproximadamente. Durante a oficina, solicitou-se aos participantes que preenchessem uma ficha informando o sexo, a idade, o curso e o semestre em que se encontravam para o mapeamento do perfil do grupo de trabalho. O material foi recolhido e acondicionado de modo a manter o sigilo sobre a identidade de cada participante.

Os encontros assumiram o formato de uma apresentação de powerpoint integrada com atividades baseadas na elaboração de trabalhos científicos. Os tópicos abordados e seus objetivos são explicitados na Tabela 1.

Tabela 1. Tópicos e objetivos abordados na oficina de Elaboração de Trabalhos Científicos

\section{Tópico/atividade}

Definições básicas

Vídeo sobre citações

Modalidades de trabalho científico

Análise de dois resumos simples

Escrita científica

Dicas de leitura

\section{Objetivo}

Apresentar aos participantes a estrutura básica de um trabalho científico (introdução, objetivos, método, resultados, discussão, conclusão, referências) e explicar de quais informações se compõe cada seção do trabalho.

Explicar aos participantes as diferenças entre citação direta e citação indireta. Explicar aos participantes as diferenças entre resumo simples, resumo expandido, trabalho completo e artigo.

Mostrar aos participantes como a forma de apresentar as informações no trabalho pode contribuir para a qualidade do texto ou para prejudicá-lo. Apresentar dicas sobre escrita científica. Indicar materiais de apoio aos participantes. 
A pesquisa

científica pode

ser um excelente

instrumento

educativo na

medida em que

leva os discentes

a lidar com o

processo de

construção do

conhecimento, e

não apenas com

o resultado desse

processo (Massi \&

Queiroz, 2010).
Os dispositivos apresentaram os conteúdos mais relevantes sobre a elaboração dos diferentes tipos de comunicação científica e a forma como os participantes poderiam organizar melhor as informações de um estudo (em introdução, método, resultados, discussão, conclusão e referências). Além disso, foi utilizado um vídeo sobre normas e citações que possibilitou abordar aspectos importantes sobre a escrita de textos científicos de forma clara e não cansativa. Considera-se que o vídeo auxiliou na troca de informações, oferecendo detalhes relevantes sobre esses temas. Também foi realizada uma atividade, preparada pelas coordenadoras da oficina, na qual foi proposta a análise individual de dois resumos simples e, posteriormente, uma discussão em conjunto, a fim de identificar os critérios utilizados pelos participantes para avaliar a qualidade dos trabalhos que produzem. A explicitação de critérios de análise buscou gerar uma reflexão nos participantes sobre os fatores a serem considerados durante a elaboração e a submissão de trabalhos acadêmicos.

Optou-se pela utilização desses recursos, porque os mesmos permitem trabalhar um assunto de forma mais dinâmica e sistemática, além de estimularem discussões e poderem fazer emergir dúvidas presentes em seus trabalhos cotidianos na elaboração de comunicações científicas. Ao final das atividades desenvolvidas, foram sugeridas leituras que oferecem dicas para a elaboração de trabalhos científicos, como os textos Apresentação de trabalho em eventos científicos: comunicação oral e painéis, de Carmo e Prado (2005), e Publicar em Psicologia: um enfoque para a revista científica, de Sabadini et al. (2009). Os participantes também responderam a uma ficha de avaliação com o objetivo de promover melhorias para os próximos trabalhos a serem desenvolvidos no projeto.

\section{Resultados e discussão}

A procura por cursos de pós-graduação tem aumentado diariamente face à competitividade atual do mercado de trabalho, com um crescente número de profissionais que buscam atualizações técnicas e de formação quanto a sua profissão (Teixeira, 2002). Por essa razão, optou-se por integrar a oficina de Elaboração de Trabalhos Científicos a esse projeto realizado com estudantes universitários, uma vez que as atividades desenvolvidas na pesquisa e na extensão durante o curso facilitam a permanência no mundo acadêmico para aqueles alunos que se interessam em continuar estudando após a graduação. Além disso, esse tipo de atividade frequentemente é percebido como uma possibilidade adicional de estabelecer contatos mais estreitos com professores, o que pode facilitar a inserção profissional de diferentes maneiras (Teixeira \& Gomes, 2004).

Somado a isso, tem-se enfatizado a ideia de que os estudantes aprendem com mais propriedade a sua profissão através da participação em atividades de pesquisa. Afinal, elas colocam os alunos em contato direto com problemas do cotidiano profissional, o que os estimula a propor soluções para os desafios que se apresentam (Freire, Reis, \& Verenguer, 2002). A pesquisa científica pode ser um excelente instrumento educativo na medida em que leva os discentes a lidar com o processo de construção do conhecimento, e não apenas com o resultado desse processo (Massi \& Queiroz, 2010). Portanto, participar de atividades de pesquisa é importante não somente para aqueles que pretendem seguir carreira acadêmica mas também para os estudantes que pretendem enfrentar o mercado de trabalho, já que lhes garante maior conhecimento sobre as práticas profissionais de sua área. 
Não foram apenas alunos da graduação que procuraram as oficinas oferecidas sobre a temática. Estavam presentes também alunos de diversos cursos de pós-graduação (especialização, mestrado e doutorado) e de cursos técnicos oferecidos pela universidade. A presença desse público nos encontros realizados pode ser compreendida pela necessidade de publicar trabalhos qualificados e em quantidade, aspecto central para ascensão e êxito de um pesquisador ou profissional atualmente. Esse cenário estimula os atores envolvidos com o meio acadêmico a respeitarem os rigorosos padrões da escrita científica, já que o discurso científico possui normas próprias, de maneira que publicar em uma revista ou apresentar seu trabalho em um evento implica enquadrar seus pensamentos em um modelo preestabelecido, que normatiza formas de apresentar as ideias (Sabadini et al., 2009).

A publicação implica ainda a disseminação do conhecimento e, em consequência, os autores permitem o acesso às suas descobertas e produções. Assim, há uma grande troca de ideias e de experiências, principalmente na era atual, em que as informações são compartilhadas de forma mais rápida e prática. Diante disso, a busca por escrever bem e de maneira adequada para o meio acadêmico tem sido cada vez maior (Sabadini et al., 2009). Tais fatos podem justificar o elevado número de acadêmicos que buscaram informações sobre essas oficinas.

A quantidade de interessados e de participantes das oficinas surpreendeu positivamente os coordenadores. Acreditavase que a maioria dos estudantes daria maior ênfase ao momento da apresentação dos trabalhos, preocupando-se com a necessidade de falar em público e não com a fase de escrita e de elaboração do trabalho propriamente dito. Contudo, foi possível perceber que esse tema atraiu os estudantes, já que eles se mostraram participativos durante todos os encontros realizados sobre esse assunto.

Os participantes das oficinas pareciam desejar mais apreender informações do que compartilhar experiências, porém sempre respondiam às questões apresentadas pelos coordenadores. Além disso, possuíam diversas dúvidas, principalmente sobre questões pontuais, como a diferença entre os termos referências e referências bibliográficas, a classificação de resumos em simples ou expandido, de que forma se elabora um problema de pesquisa e onde é possível encontrar as normas da ABNT (ou de outros sistemas de referenciação).

Os discentes também perguntaram sobre metodologia, buscando compreender qual método seria mais adequado para determinado estudo, e sobre como acessar o Portal de periódicos da CAPES. Tais questões não puderam ser devidamente explicitadas nessa oficina devido ao seu tempo de duração. Por isso, foi sugerido que os estudantes entrassem em contato com seus orientadores para sanar essas dificuldades, pois entende-se que as pesquisas de cada área possuem características específicas que podem ser mais bem compreendidas e definidas pelos investigadores do campo. No que se refere ao acesso ao Portal CAPES, foi brevemente explicado e anotado como um possível tópico para ser trabalhado em próximas oficinas a serem ofertadas sobre elaboração de trabalhos científicos. Contudo, as dúvidas relativas ao acesso ao Portal CAPES indicam a necessidade de refletir sobre as práticas de Iniciação Científica vigentes na(s) universidade(s). Acredita-se que a promoção de cursos que tratem do tema acesso ao Portal CAPES possa ser uma das atividades de base dos grupos de pesquisa, especialmente para novos integrantes, uma vez que esse Portal detém informações importantes de acesso restrito. Durante o trabalho realizado 
nas oficinas, porém, identificou-se que nem todos participavam de grupos de pesquisa. Assim, é imprescindível que esse assunto seja abordado tanto nas disciplinas de metodologia, ofertadas pela maioria dos cursos de graduação e de pós-graduação da universidade, como em cursos de extensão específicos, como os promovidos pela própria CAPES para divulgação desse portal.

Assim, muitas das perguntas formuladas pelos participantes demonstram que ainda hoje estão presentes dificuldades em relacionar ensino e pesquisa nas disciplinas, uma vez que alguns professores estabelecem diferenças entre a sala de aula e o espaço de pesquisa. Na maioria dos casos, a graduação continua sendo dedicada à reprodução, e não à produção de conhecimentos, enquanto os espaços de pesquisa, principalmente as atividades vinculadas aos grupos de pesquisa, são muito mais valorizados, prevendose a mudança de comportamento dos professores, que passariam a estimular mais a busca de conhecimento por parte dos alunos envolvidos em tais atividades (Massi \& Queiroz, 2010).

De maneira geral, o grupo de participantes das oficinas pareceu gostar da atividade prática realizada, na qual os estudantes foram convidados a analisar dois resumos simples (um considerado bem elaborado e outro mal elaborado). Essa tarefa buscou promover a reflexão e ilustrar os assuntos trabalhados. Os estudantes conseguiram identificar as partes essenciais do trabalho (introdução, objetivo, método, resultados e conclusão) com maior facilidade no resumo considerado bem elaborado, e perceberam que essa clareza pode diferenciar os trabalhos que escrevem; ademais, demonstraram interesse nas dicas concedidas pelos coordenadores para melhorar a clareza do texto, por exemplo, utilizar as frases na ordem direta para auxiliar o fluxo da leitura, evitar citações literais, pois podem demonstrar falta de conhecimento sobre o conteúdo estudado, quando escrever os números por extenso e quando citá-los na forma numérica, não elaborar títulos longos (normalmente, títulos com mais de 15 palavras são considerados extensos), usar verbos no infinitivo para apresentar os objetivos do estudo, usar verbos no tempo pretérito para descrever os resultados, indicar as principais contribuições do trabalho e quais suas limitações, sugerindo novos estudos na sessão das considerações finais ou das conclusões, identificar a diferença entre os termos considerações finais e conclusão e entre os termos método e metodologia, utilizar os termos corretos para cada tipo de pesquisa, como dados e sujeito, em se tratando de pesquisas de natureza quantitativa, e informações e respondente para pesquisas qualitativas.

Essas orientações costumam ser valorizadas por estudantes universitários, já que necessitam aprender as diferenças entre a forma como realizavam as leituras e a escrita do ensino médio e o modo como devem fazê-lo na universidade. A elaboração de um texto no ensino superior requer a leitura de várias fontes, nas quais diferentes autores apresentam posturas distintas sobre um mesmo assunto. Já os textos lidos no ensino médio, frequentemente, apresentam uma única posição, considerada absoluta. Também é possível que os alunos desse nível de ensino respondam perguntas sobre a leitura de um texto sem compreendê-lo, dado que não se requer a construção de uma resposta além da reprodução dos dados (Carlino, 2007). Dessa forma, os discentes universitários podem perceber que escrever não envolve somente questões de gramática e ortografia mas também a estruturação e a expressão de ideias.

As fichas de avaliação que foram preenchidas pelos participantes ao final da oficina possibilitaram identificar o nível de satisfação dos estudantes de forma mais objetiva. 
Observou-se que as oficinas contemplaram plenamente as suas expectativas. As atividades propostas, a utilização dos recursos materiais e a indicação de leituras foram consideradas adequadas e percebidas como facilitadoras do processo de aprendizagem, e a maioria dos alunos enfatizou que participaria de outra oficina oferecida pelo projeto. Por fim, ressalta-se que a primeira oficina foi considerada ótima ou boa por grande parte dos discentes. A cada nova oficina promovida, aumentou o número de acadêmicos que avaliaram o encontro como ótimo, o que pode ser um indicador da elevação da qualidade das oficinas, demonstrando o desenvolvimento e a apropriação dos coordenadores sobre o assunto.

\section{Considerações finais}

O objetivo deste artigo foi apresentar a experiência de três oficinas sobre elaboração de trabalhos científicos e refletir sobre as principais dúvidas manifestadas pelos participantes. As oficinas realizadas através desse projeto tem constituído um espaço para discutir e até mesmo para aprender habilidades fundamentais para o desenvolvimento de carreira de estudantes universitários.

O alto índice de procura dos discentes pela oficina de Elaboração de Trabalhos Científicos reflete o reconhecimento da importância dessa prática por parte dos alunos da graduação e da pós-graduação e aponta lacunas na formação desses indivíduos. As dúvidas manifestadas pelos participantes suscitam questões sobre quais tópicos têm sido trabalhados nas disciplinas de metodologia da pesquisa dos diversos cursos de graduação. Questionase também se e de que forma os grupos de pesquisa têm abordado esse tema ou ainda se acadêmicos de Iniciação Científica, mestrandos e doutorandos têm recebido instruções básicas de seus orientadores para a produção científica. Afinal, supõe-se que grande parte dos cursos ofereça a disciplina Metodologia da Pesquisa e que estudantes da pós-graduação possuam instruções mínimas para produzir junto a seus orientadores. $\mathrm{O}$ objetivo desta discussão não é identificar responsáveis, mas favorecer a reflexão sobre o papel de professores na transmissão e de discentes na busca por essas informações. Pensa-se que as universidades poderiam ampliar os espaços para que esse tipo de conhecimento fosse construído. Nesse sentido, os alunos poderiam beneficiar-se tanto de oportunidades tradicionais, como aulas, grupos de estudo e orientações em pesquisa, quanto de ações inovadoras, como é o exemplo dessa oficina.

As oficinas apresentam algumas limitações que devem ser mencionadas. Uma delas refere-se ao local onde são realizadas, no campus da universidade, que se localiza em um bairro afastado do centro da cidade. A maioria dos cursos é sediada no mesmo campus, contudo, há outros que se localizam no centro. Assim, estudantes desses cursos podem encontrar dificuldades para se deslocar até o campus, especialmente aqueles que trabalham durante o dia e estudam à noite. Ademais, a universidade oferece cursos em outros Municípios, o que também dificulta o acesso desses alunos. Outra limitação corresponde aos tópicos abordados. Optou-se por trabalhar com questões básicas, pois não se sabe qual o conhecimento prévio dos participantes quando se inscrevem. A heterogeneidade das áreas de estudo dos discentes também interfere nesse aspecto. Sabe-se que pesquisas de áreas diferentes possuem particularidades que são desconhecidas por estudiosos de outros campos. Dessa forma, torna-se inviável para os coordenadores responderem dúvidas específicas. Nesses casos, sugere-se que os acadêmicos consultem o professor orientador.

Acredita-se que as limitações encontradas 
devam indicar caminhos para o aperfeiçoamento das atividades realizadas. Então, pensa-se em oferecer as oficinas do projeto diretamente às coordenações dos cursos de graduação para diminuir os possíveis prejuízos relacionados ao local onde as oficinas são desenvolvidas e ao nível de complexidade dos tópicos abordados. Assim, é possível ajustar o horário, o local e o conteúdo do encontro à demanda apresentada por determinado grupo de estudantes.

Não se pode afirmar o quanto do conteúdo da oficina foi apreendido pelos participantes e até que ponto os tópicos discutidos serão, de fato, utilizados. Contudo, acredita-se que o interesse em participar do encontro, em pensar e em questionar diversos aspectos da produção científica sinalize o desejo dos estudantes já envolvidos com pesquisa de melhorar a qualidade das publicações ou os comportamentos exploratórios dos alunos que ainda não tiveram essa experiência.

É possível que outras universidades também ofereçam esse tipo de atividade para o desenvolvimento de habilidades dos acadêmicos, mas os relatos encontrados não caracterizavam estudantes universitários como público-alvo. Então, espera-se que este artigo estimule a criação de propostas semelhantes em outras instituições, bem como publicações de experiências semelhantes. 
Clarissa Tochetto de Oliveira

Doutoranda do Programa de Pós Graduação em Psicologia da Universidade Federal do Rio Grande do Sul, Porto Alegre - RS - Brasil.

E-mail: clarissa.tochetto@gmail.com

\section{Anelise Schaurich dos Santos}

Mestranda do Programa de Pós-graduação em Psicologia da Universidade Federal de Santa Maria, Santa Maria RS - Brasil.

E-mail: anelise_ssantos@hotmail.com

\section{Danielle da Costa Souto}

Discente do Programa de Pós-Graduação em Psicologia: Ênfase em Psicologia da Saúde e pesquisadora na Universidade Federal de Santa Maria, Santa Maria - RS - Brasil.

E-mail: daniellessouto@hotmail.com

\section{Ana Cristina Garcia Dias}

Doutora em Psicologia Escolar e do Desenvolvimento Humano pela Universidade de São Paulo e Professora Adjunta da Universidade Federal de Santa Maria, Santa Maria - RS - Brasil.

E-mail: anacristinagarciadias@gmail.com

Endereço para envio de correspondência:

Rua Porto Verde, 185, Bairro Camobi. CEP: 9110-590. Santa Maria, RS.

Recebido 19/03/2013, 1a Reformulação 05/09/2013, Aprovado 16/10/2013. 


\section{Referências}

Bardagi, M. P., Lassance, M. C. P., \& Paradiso, A. C. (2003). Trajetória acadêmica e satisfação com a escolha profissional de universitários em meio de curso. Revista Brasileira de Orientação Profissional, 4(1), 153-166.

Bardagi, M. P., Lassance, M. C., Paradiso, A., \& Menezes, I. (2006). Escolha profissional e inserção no mercado de trabalho. Revista Semestral da Associação Brasileira de Psicologia Escolar e Educacional (ABRAPEE), 10(1), 69-82.

Carlino, P. (2007). ¿Qué nos dicen las investigaciones internacionales sobre la escritura en la universidad? Cuadernos de Psicopedagogía, 4, 21-40.

Carmo, J. S., \& Prado, P. S. T. (2005). Apresentação de trabalho em eventos científicos: comunicação oral e painéis. Interação em Psicologia, 9(1), 131-142.

Conselho Nacional de Desenvolvimento Científico e Tecnológico. (CNPq). (2002). Regimento interno. Recuperado em 22 fev., 2013, de http://cnpq.br/web/guest/regimento-interno

Freire, E. S., Reis, M. C. C., \& Verenguer, R. C. (2002). Educação física: pensando a profissão e a preparação profissional. Revista Mackenzie de Educação Física e Esporte, 1(1), 39-46.

Giordani, E. M., Mendes, A. M. M., \& Henriques, C. (2007). Iniciação científica e pedagogia universitária. In Anais do $V$ Seminário Nacional de Pedagogia Universitária (pp. 1-6). São Leopoldo, RS: Unisinos.

Hurtado, S., Eagan, M. K., Tran, M. C., Newman, C. B., Chang, M. J., \& Velasco, P. (2011). "We do science here": Underrepresented students' interactions with faculty in different college contexts. Journal of Social Issues, 67(3), 553579. doi: $10.1111 / j .1540-4560.2011 .01714 . x$

Kellogg, R. T., \& Raulerson, B. A. (2007). Improving the writing skills of college students. Psychonomic Bulletin \& Review, 14(2), 237-242.

Machado, A. M. N. (2002). A relação entre a autoria e a orientação no processo de elaboração de teses e dissertações. In L. Bianchetti \& A. M. N. Machado (Orgs.), A bússola do escrever: desafios e estratégias na orientação de teses e dissertações (pp. 45-65). São Paulo: Cortez.

Maia, R. T. (2008). A importância da disciplina de metodologia científica no desenvolvimento de produções acadêmicas de qualidade no nível superior. Revista Urutágua, 14(1), 1-8.

Massi, L., \& Queiroz, S. L. (2010). Estudos sobre iniciação científica no Brasil: uma revisão. Cadernos de Pesquisa, 40(139), 173-197.

Nahas, F. X., Ferreira, L. M., Neto, M. S., \& Garcia, E. B. (2004). Elaboração de trabalho científico. Revista da Sociedade Brasileira de Cirurgia Plástica, 19(2), 11-28.

Petroianu, A. (2002). Autoria de um trabalho científico. Revista da Associação de Medicina Brasileira, 48(1), 60-65. doi: http:// dx.doi.org/10.1590/S0104-42302002000400029

Reis, L. A. (2007). Programa institucional de bolsas de iniciação científica do IBAMA: uma política de pesquisa. Dissertação de mestrado. Universidade Católica de Brasília, Brasília, DF.

Sabadini, A. A. Z. P., Sampaio, M. I. C., \& Koller, S. H. (2009). Publicar em psicologia: um enfoque para a revista científica. São Paulo: Associação Brasileira de Editores Científicos de Psicologia/Instituto de Psicologia da Universidade de São Paulo.

Teixeira, M. A. P. (2002). A experiência de transição entre a universidade e o mercado de trabalho na adultez jovem. Tese de doutorado. Universidade Federal do Rio Grande do Sul, Porto Alegre, RS.

Teixeira, M. A. P., \& Gomes, W. B. (2004). Estou me formando... e agora?: Reflexões e perspectivas de jovens formandos universitários. Revista Brasileira de Orientação Profissional, 5(1), 47-62. 